\title{
Verifiable Control of a Swarm of Unmanned Aerial Vehicles
}

\author{
D. J. Bennet and C. R. McInnes \\ Department of Mechanical Engineering \\ University of Strathclyde \\ Glasgow, UK
}

\begin{abstract}
This paper considers the distributed control of a swarm of unmanned aerial vehicles investigating autonomous pattern formation and reconfigurability. A behaviour-based approach to formation control is considered with a velocity field control algorithm developed through bifurcating potential fields. This new approach extends previous research into pattern formation using potential field theory by considering the use of bifurcation theory as a means of reconfiguring a swarm pattern through a free parameter change. The advantage of this kind of system is that it is extremely robust to individual failures, is scalable and also flexible. The potential field consists of a steering and repulsive term with the bifurcation of the steering potential resulting in a change of the swarm pattern. The repulsive potential ensures collision avoidance and an equally spaced final formation. The stability of the system is demonstrated to ensure that desired behaviours always occur, assuming that at large separation distances the repulsive potential can be neglected through a scale separation that exists between the steering and repulsive potential. The control laws developed are applied to a formation of 10 unmanned aerial vehicles using a velocity field tracking approach, where it is shown numerically that desired patterns can be formed safely ensuring collision avoidance.
\end{abstract}




\section{NOMENCLATURE}

$\begin{array}{ll}N & \text { number of UAVs in formation } \\ U & \text { artificial potential field } \\ U^{S} & \text { steering potential } \\ U^{R} & \text { repulsive potential } \\ \mu & \text { bifurcation parameter } \\ C_{r}, L_{r} & \text { repulsive potential amplitude and length scale } \\ \mathbf{x} & \text { UAV position vector } \\ \mathbf{v} & \text { UAV velocity vector } \\ \alpha & \text { amplitude of dissipative term } \\ \mathbf{J} & \text { Jacobian } \\ L & \text { Lyapunov function } \\ V & \text { UAV speed (m/s) } \\ \dot{V} & \text { UAV acceleration }\left(m / s^{2}\right) \\ \psi & \text { UAV heading angle (radians) } \\ \dot{\psi} & \text { UAV turn rate (radians } / \mathrm{s}) \\ \lambda_{v}, \lambda_{\psi} & \text { speed and heading angle inverse time constants } \\ Z_{r} & \text { repulsive potential sensing radius } \\ g & \text { gravitational constant, } 9.81 m s^{-2} \\ u_{c} & \text { desired cruise speed of } \mathrm{UAV}, 2.7 \mathrm{~ms}^{-1} \\ t & \text { time (s) } \\ & \end{array}$

\section{INTRODUCTION}

Interest in unmanned aerial vehicles (UAVs) has grown in recent years, with a variety of civil and military applications such as scientific data gathering, military reconnaissance and convoy protection $[1,2,3,4,5,6]$. In addition to the development of single UAV systems, it has been shown that having multiple 
UAVs flying in formation will allow for applications, such as interferometric imaging, that could not be achieved through single UAVs [7]. As the number of UAVs increase, controlling the system in a centralised way becomes unrealistic, so that decentralised control methods have been developed to overcome this problem.

In the area of distributed multi-vehicle systems some work is motivated by the emergent and self-organised behaviour that is seen in nature. Through simple local interactions a school of fish or flock of birds, for example, will aggregate together to form global emergent behaviour [7]. Brooks [8] introduced the concept of a behavioural control architecture taking inspiration from natural behaviours. By having this form of control we can have a system that, although controlled through relatively simple laws, will achieve a desired behaviour and have the advantages of being a scalable, robust and flexible system [9].

Artificial potential fields are an example of behavioural control architecture $[7,10,11]$ and were first introduced by Khatib [12] in the area of obstacle avoidance for manipulators. More recently they have been applied successfully in the area of autonomous robot motion planning $[13,14]$ and in space applications $[15,16,17]$. The basic idea behind artificial potential fields is to create a workspace where each UAV is attracted towards a goal state with a repulsive potential ensuring collide avoidance [14]. As the UAV swarm may be required to achieve different tasks, a desirable property of the system would be reconfigurability. In order to minimise computational expense bifurcation theory can be used to reconfigure the formation through a simple free parameter change.

Other approaches to the control of UAVs include Reynolds flocking theory [?] that takes a set of rules and applies them to all the vehicles in the group. Reynolds was able to successfully show the first computer simulation of flocking 
agents and several authors have since extended his theory and applied it specifically to control a swarm of UAVs $[18,19]$. Another approach to UAV control is graph theory that represents the local interactions and spatial distribution of a swarm of UAVs in a directed graph [20]. The virtual structure approach is also used that treats each UAV as a particle that attempts to maintain a fixed geometric relationship [7, 21].

The purpose of this paper is to investigate the distributed control of pattern formation and reconfigurability in a swarm of UAVs. A behavioural control architecture is developed through the artificial potential field method and bifurcation theory that allows for the creation of autonomous swarm patterns that can be altered through manipulation of the free parameters of the potential field. This new approach consists of a steering and repulsive potential field with the bifurcation of the steering potential resulting in the formation of different patterns. The repulsive potential ensures collision avoidance and an equally spaced final formation. The advantages of this system are that it is robust, scalable and flexible. In addition, for real safety critical applications it is essential that the stability of the system is ensured. As opposed to algorithm validation this paper mathematically proves the stability of the system. It is shown that there exists a scale separation between the steering and repulsive potential so that UAV system moves under the influence of a far field steering potential but with short range collisions. It can then be proven analytically that the desired behaviours always occur. The model is then applied to a velocity field tracking approach that generates a set of commands to control the UAV heading and speed.

The paper proceeds as follows. In section 2 we describe the model used and explain the artificial potential field method and bifurcation theory. We also discuss the linear and non-linear stability of the models developed. Section 3 
shows the numerical results of simulations demonstrating pattern formations and reconfigurability. In section 4 we consider a swarm of 10 UAVs desired to form a double ring pattern and then bifurcate into two different ring patterns, traveling at constant speed and ensuring collision avoidance throughout the simulation.

\section{FORMATION MODEL}

\subsection{Model}

We consider a swarm of homogeneous UAVs $(1 \leq i \leq N)$ described through a second-order dynamical system as shown in Eq. 1 and 2. The negative gradient of an artificial potential function, $U$, describes a virtual force acting on each UAV with mass, $m$, position, $\mathbf{x}_{i}$, and velocity, $\mathbf{v}_{i}$;

$$
\begin{gathered}
\frac{d \mathbf{x}_{i}}{d t}=\mathbf{v}_{i} \\
\frac{d \mathbf{v}_{i}}{d t}=-\nabla_{i} U^{S}\left(\mathbf{x}_{i}\right)-\nabla_{i} U^{R}\left(\mathbf{x}_{i j}\right)-\sigma \mathbf{v}_{i}
\end{gathered}
$$

It can be seen from Eq. 2 that the virtual force experienced by each UAV is dependent upon two artificial potential functions and a dissipative term, where $\sigma$ controls the amplitude of this dissipation. The first term in Eq. 2 is defined as the steering potential, $U^{S}$, and is used to command each UAV to a desired position with the repulsive potential, $U^{R}$, ensuring collision avoidance and an equally spaced formation.

The repulsive potential is a simple pairwise exponential function that is based on a generalized Morse potential [22] as shown in Eq. 3;

$$
U_{i j}^{R}=\sum_{j, j \neq i} C_{r} \exp ^{-\left|\mathbf{x}_{i j}\right| / L_{r}}
$$


where $C_{r}$ and $L_{r}$ represent the amplitude and length-scale of repulsive potential respectively and $\left|\mathbf{x}_{i j}\right|=\left|\mathbf{x}_{i}-\mathbf{x}_{j}\right|$.

The total repulsive force on the $i^{t h} \mathrm{UAV}$ is dependent upon the position of all the other $(N-1)$ UAVs in the formation. The repulsive potential is therefore used to ensure that as the UAVs are steered towards the goal state they do not collide with each other. Once all the UAVs have been driven to the desired equilibrium state the repulsive potential also ensures that they are equally spaced for symmetric formations.

\subsection{Artificial Potential Function Scale Separation}

As noted in the previous section the dynamics of each UAV is dependent upon the gradient of two different artificial potential functions. The steering and repulsive potential are a function of position only with length scale $R$ and $L_{r}$ respectively as shown in Eq. 4 and 5;

$$
\begin{gathered}
U^{S}=f(X, R) \\
U^{R}=C_{r} \exp ^{-X / L_{r}}
\end{gathered}
$$

For illustration we consider a simple 1-dimensional system with position coordinate $X$.

Defining an outer region dependent upon the steering potential only and an inner region dependent upon the repulsive potential only we can show that these two regions are separated so that each UAV moves under the influence of the long-range steering potential but with short range collisions (for $L_{r} / R<<1$ ) effectively creating a boundary layer between them. This can then be used 
to determine the non-linear stability properties of the system considering the steering potential only.

For 1D motion of a UAV of mass $m$ and damping constant $\sigma$ we have;

$$
m \frac{d V}{d t}=-\frac{d U^{R}}{\partial X}-\frac{d U^{S}}{d X}-\sigma V
$$

so that,

$$
m V \frac{d V}{d X}=\frac{C_{r}}{L_{r}} \exp ^{-X / L_{r}}-\frac{d U^{S}}{d X}-\sigma V
$$

Scaling $X$ such that $S=X / R$ then;

$$
\frac{1}{R} m V \frac{d V}{d S}=\frac{C_{r}}{L_{r}} \exp ^{-\frac{R}{L_{r}} S}-\frac{1}{R} \frac{d U^{S}}{d S}-\sigma V
$$

Now define $\varepsilon=\frac{L_{r}}{R}<<1$ so that;

$$
m V \frac{d V}{d S}=\frac{C_{r}}{\varepsilon} \exp -\frac{S}{\varepsilon}-\frac{d U^{S}}{d S}-\sigma R V
$$

Let $\varepsilon \rightarrow 0$ in order to consider 'far field' dynamics which forms a singularly perturbed system;

$$
\lim _{\varepsilon \rightarrow 0} \frac{1}{\varepsilon} \exp ^{(-S / \varepsilon)}=0
$$

Therefore at large separation distances the repulsive potential vanishes and we can consider the steering potential only when considering the stability of analysis of the system.

Conversely if we define $\bar{S}=\frac{S}{\varepsilon}$ we find that the 'near field' dynamics are defined by; 


$$
m V \frac{d V}{d \bar{S}}=C_{r} \exp ^{-\bar{S}}-\varepsilon R\left(\frac{1}{L_{r}} \frac{d U^{S}}{d \bar{S}}+\sigma V\right)
$$

and letting $\varepsilon \rightarrow 0$;

$$
m V \frac{d V}{d \bar{S}}=C_{r} \exp ^{-\bar{S}}
$$

Thus, at small separations the steering potential vanishes. If we then consider the $2^{\text {nd }}$ order system and assume that $m / \sigma<<1$ so that the dynamics are overdamped, we obtain a velocity field defined as shown in Eq. 15.

$$
\frac{m}{\sigma} V \frac{d V}{d \bar{S}}=-\frac{1}{\sigma} \nabla_{i} U^{S}(X)-\frac{1}{\sigma} \nabla_{i} U^{R}(X)-V
$$

assuming that $m / \sigma<<1$ so that the system is overdamped we find that,

$$
-\frac{1}{\sigma} \nabla_{i} U^{S}(X)-\frac{1}{\sigma} \nabla_{i} U^{R}(X)-V=0
$$

thus,

$$
\frac{d X}{d t}=-\frac{1}{\sigma} \nabla_{i} U^{S}(X)-\frac{1}{\sigma} \nabla_{i} U^{R}(X)
$$

Each UAV therefore acts under the influence of a long range steering potential but with short range collisions, allowing us to treat the collisions separate in the subsequent stability analysis. We can also now use the first-order velocity field to control the formation of UAVs as shown in Eq. 16;

$$
\frac{d \mathbf{x}_{i}}{d t}=-\nabla_{i} U^{S}\left(\mathbf{x}_{i}\right)-\nabla_{i} U^{R}\left(\mathbf{x}_{i j}\right)
$$

By assuming that the second-order system is overdamped the UAVs will closely track this velocity field. 


\subsection{Steering Potential - 1 parameter static bifurcation}

Referring to Eq. 16 we can base the steering potential on a supercritical pitchfork bifurcation [23] as shown in the first two terms of Eq. 17. This potential drives each UAV to a goal distance, $r$, from the origin in the $x-y$ plane with the last term in Eq. 17 ensuring that the formation is created in the $x-y$ plane, where $\alpha$ controls the amplitude of this quadratic potential;

$$
U^{S}\left(\mathbf{x}_{i} ; \mu, \alpha\right)=-\frac{1}{2} \mu\left(\rho_{i}-r\right)^{2}+\frac{1}{4}\left(\rho_{i}-r\right)^{4}+\alpha z_{i}^{2}
$$

where cylindrical polar coordinates, $\mathbf{x}_{i}=\left(\rho_{i}, z_{i}\right)^{T}$, are used, neglecting the $\theta$ term as the potential field is rotationally symmetric.

Depending upon the sign of $\mu$, the steering potential can have two distinct forms. Figure 1 shows the shape of the potential and the corresponding velocity field when $\mu<0$ and $\mu>0$. Figure 2 shows the bifurcation diagram for the steering potential indicating a bifurcation from a single local minimum into two local minima when $\mu=0$. 


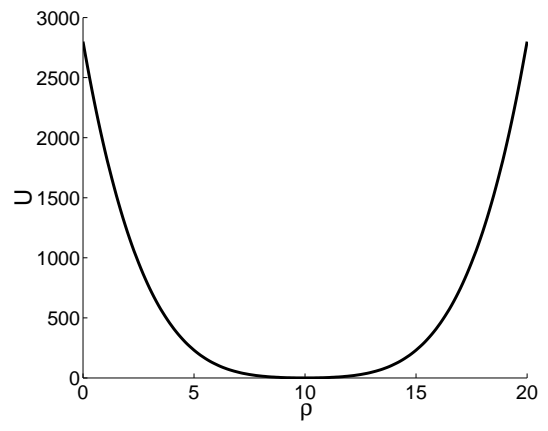

(i)

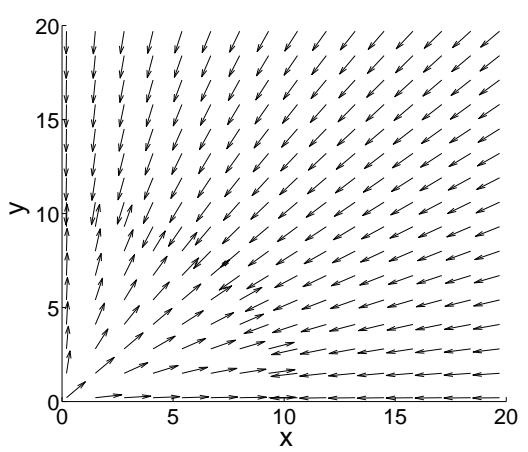

(iii)

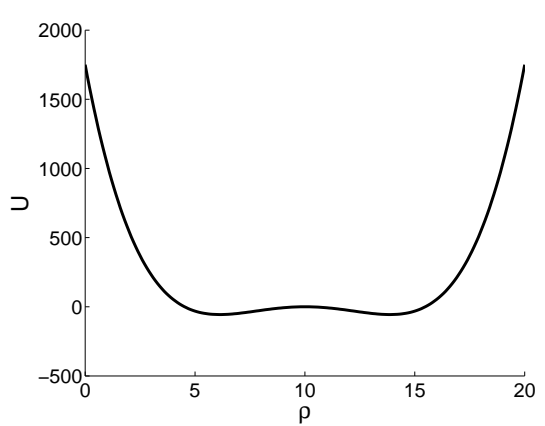

(ii)

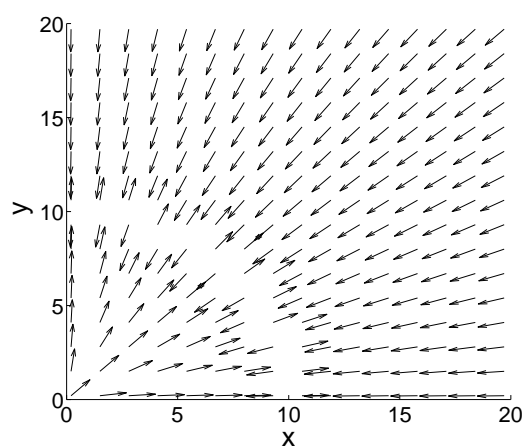

(iv)

Figure 1: Potential and velocity fields (i) potential $\mu<0$ (ii) potential $\mu>0$

(iii) velocity field $\mu<0$ (iv) velocity field $\mu>0$

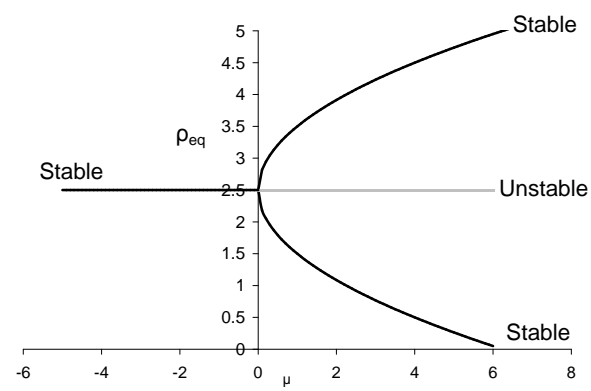

Figure 2: Supercritical pitchfork bifurcation diagram 
The equilibrium states of the potential occur whenever $\partial U / \partial \rho_{i}=0$ and $\partial U / \partial z_{i}=$ 0 . Therefore;

$$
\begin{gathered}
\frac{\partial U}{\partial \rho_{i}}=-\mu\left(\rho_{i}-r\right)+\left(\rho_{i}-r\right)^{3} \\
\frac{\partial U}{\partial z_{i}}=\alpha z_{i}
\end{gathered}
$$

If $\mu \leq 0$ equilibrium occurs when $\rho_{i}=r$. If $\mu>0$ equilibrium occurs when $\rho_{i}=r, r \pm \sqrt{\mu}$. Therefore, a single ring will bifurcate to a double ring using $\mu$ as a control parameter.

For a function consisting of two variables the stability of the system is determined from the sign of the determinant of the Hessian matrix [?], $D$, given in Eq. 20;

$$
D=\frac{\partial^{2} U}{\partial \rho_{i}^{2}} \frac{\partial^{2} U}{\partial z_{i}^{2}}-\left[\frac{\partial^{2} U}{\partial \rho_{i} \partial z_{i}}\right]^{2}
$$

The conditions for stability are as follows;

(i) $D>0, \partial^{2} U / \partial \rho_{i}^{2}>0 \Longrightarrow$ equilibrium point is a stable minimum.

(ii) $D>0, \partial^{2} U / \partial \rho_{i}^{2}<0 \Longrightarrow$ equilibrium point is a unstable maximum.

(iii) $D<0 \Longrightarrow$ equilibrium point is a saddle.

The second derivative of the potential is shown in Eq. 21, 22 and 23;

$$
\begin{gathered}
\frac{\partial^{2} U}{\partial \rho_{i}^{2}}=-\mu+3\left(\rho_{i}-r\right)^{2} \\
\frac{\partial^{2} U}{\partial z_{i}^{2}}=\alpha \\
\frac{\partial^{2} U}{\partial \rho_{i} \partial z_{i}}=0
\end{gathered}
$$


From Eq. 22 as $\alpha$ is positive, $\partial^{2} U / \partial z_{i}^{2}>0$. From Eq. 21 it can be seen that $\partial^{2} U / \partial \rho_{i}^{2} \gtrless 0$ depending on the values of $\mu$. Therefore, the properties of the equilibrium state $\rho_{e q}$ are shown in Table 1 ;

Table 1: Stability of equilibrium states

\begin{tabular}{cccc}
\hline \hline Bifurcation & Equilibrium & $\partial^{2} U / \partial \rho_{i}^{2}$ & Stability \\
parameter, $\mu$ & position, $\rho_{e q}$ & & \\
\hline$<0$ & $r$ & $>0$ & stable minimum \\
$>0$ & $r$ & $<0$ & unstable maximum \\
& $r+\sqrt{\mu}$ & $>0$ & stable minimum \\
& $r-\sqrt{\mu}$ & $>0$ & stable minimum \\
\hline
\end{tabular}

\subsubsection{Linear stability: 1-parameter static bifurcation}

In order to determine the linear stability of a system of UAVs subject to such a 1parameter static bifurcation steering potential we perform an eigenvalue analysis on the linearized equations of motion assuming that at large separation distances the repulsive potential can be neglected through scale separation as explained in Section 2.2. The linear stability analysis will be used to determine the local behaviour of the system by calculating its eigenvalue spectrum. Therefore, the equations of motion for the model are re-cast as;

$$
\begin{aligned}
\left(\begin{array}{c}
\dot{\rho}_{i} \\
\dot{z}_{i}
\end{array}\right) & =\left(\begin{array}{c}
-\frac{\partial d U^{S}}{\partial \rho_{i}} \\
-\frac{\partial d U^{S}}{\partial z_{i}}
\end{array}\right) \\
& =\left(\begin{array}{c}
f\left(\rho_{i}, z_{i}\right) \\
g\left(\rho_{i}, z_{i}\right)
\end{array}\right)
\end{aligned}
$$

Let $\rho_{o}$ and $z_{o}$ denote fixed points with $\dot{\rho}_{i}=\dot{z}_{i}=0$ so that; 


$$
\begin{aligned}
& f\left(\rho_{o}, z_{o}\right)=0 \\
& g\left(\rho_{o}, z_{o}\right)=0
\end{aligned}
$$

Thus, $\nabla U^{S}=0$ at equilibrium. This occurs when $\rho_{o}=r$ if $\mu<0, \rho_{o}=r, r \pm \sqrt{\mu}$ if $\mu>0$ and $z_{o}=0$. Defining $\delta \rho_{i}=\rho_{i}-\rho_{o}$ and $\delta z_{i}=z_{i}-z_{o}$ and Taylor Series expanding about the fixed points to linear order the eigenvalues of system can be found using;

$$
\left(\begin{array}{c}
\delta \dot{\rho}_{i} \\
\delta \dot{z}_{i}
\end{array}\right)=\mathbf{J}\left(\begin{array}{c}
\delta \rho_{i} \\
\delta z_{i}
\end{array}\right)
$$

where,

$$
\mathbf{J}=\left.\left(\begin{array}{cc}
\frac{\partial}{\partial \rho_{i}}\left(f\left(\rho_{i}, z_{i}\right)\right) & \frac{\partial}{\partial z_{i}}\left(f\left(\rho_{i}, z_{i}\right)\right) \\
\frac{\partial}{\partial \rho_{i}}\left(g\left(\rho_{i}, z_{i}\right)\right) & \frac{\partial}{\partial z_{i}}\left(g\left(\rho_{i}, z_{i}\right)\right)
\end{array}\right)\right|_{\rho_{o}, z_{o}}
$$

The Jacobian, $\mathbf{J}$, is then a $2 x 2$ matrix given by;

$$
\mathbf{J}=\left.\left(\begin{array}{cc}
-\frac{\partial^{2} U}{\partial \rho_{i}^{2}} & 0 \\
0 & -\frac{\partial^{2} U}{\partial z_{i}^{2}}
\end{array}\right)\right|_{\rho_{o}, z_{o}}
$$

Substituting a trial exponential solution into Eq. 27 we find that;

$$
\left(\begin{array}{c}
\delta \dot{\rho}_{i} \\
\delta \dot{z}_{i}
\end{array}\right)=\left(\begin{array}{c}
\delta \rho_{o} \\
\delta z_{o}
\end{array}\right) e^{\lambda t}
$$

Therefore, the eigenvalues, $\lambda$, of the system are found from $\operatorname{det}(\mathbf{J}-\lambda \mathbf{I})=0$.

As shown previously, if $\mu<0$ equilibrium of the system occurs when $\mathbf{x}_{o}=(r, 0)$. Evaluating the Jacobian matrix given in Eq. 29 we find that;

$$
\mathbf{J}=\left(\begin{array}{cc}
\mu & 0 \\
0 & -\alpha
\end{array}\right)
$$


The corresponding eigenvalue spectrum is therefore;

$$
\lambda_{1,2}=-\alpha, \mu
$$

As $\alpha>0$ and $\mu<0$ the eigenvalues are always negative real and the equilibrium position can therefore be considered as linearly stable.

If $\mu>0$ equilibrium of the system occurs when $\mathbf{x}_{o 1}=(r, 0), \mathbf{x}_{o 2}=(r+\sqrt{\mu}, 0)$ and $\mathbf{x}_{o 3}=(r-\sqrt{\mu}, 0)$. The Jacobian matrix evaluated at the three different equilibrium positions is given by Eq. 33, 34 and 35 respectively as;

$$
\begin{gathered}
\mathbf{J}_{1}=\left(\begin{array}{cc}
\mu & 0 \\
0 & -\alpha
\end{array}\right) \\
\mathbf{J}_{2}=\left(\begin{array}{cc}
-2 \mu & 0 \\
0 & -\alpha
\end{array}\right) \\
\mathbf{J}_{3}=\left(\begin{array}{cc}
-2 \mu & 0 \\
0 & -\alpha
\end{array}\right)
\end{gathered}
$$

The eigenvalues for $\mathbf{J}_{1}$ are;

$$
\lambda_{1,2}=-\alpha, \mu
$$

Considering the pair of eigenvalues in Eq. 36, as $\alpha>0$ and $\mu>0$ we have one positive eigenvalue so that the equilibrium position is therefore always linearly unstable.

The eigenvalues for $\mathbf{J}_{2}$ and $\mathbf{J}_{3}$ are;

$$
\lambda_{1,2}=-\alpha,-2 \mu
$$

Similarly again as $\alpha>0$ and $\mu>0$ the eigenvalues are always negative real so that these two equilibrium positions can therefore be considered as linearly 
stable.

Figure 3 confirms the linearised stability results showing the phase plane plot for $\mu<0$ and $\mu>0$.

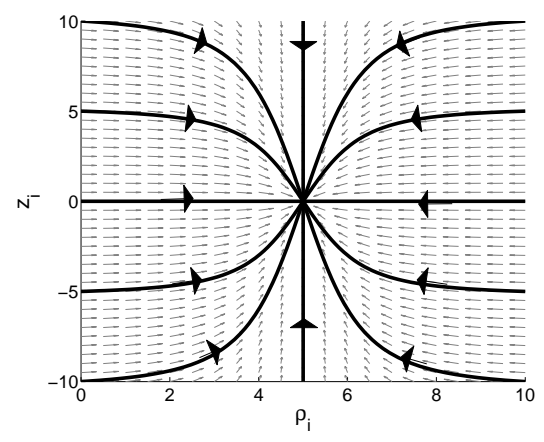

(i)

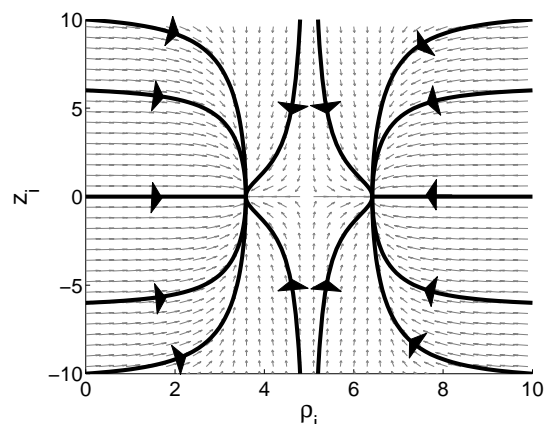

(ii)

Figure 3: Phase diagram (i) $\mu<0, r=5$ (ii) $\mu>0, r=5$

From Fig. 3(i) it can be seen that when $\mu<0$ we have one stable equilibrium position when $\mathbf{x}_{o}=(r, 0)$ as indicated by the eigenvalues given in Eq. 31. If the system then bifurcates so that $\mu>0$, the stable position at $\mathbf{x}_{o 1}=(r, 0)$ becomes unstable and positions $\mathbf{x}_{o 2}=(r+\sqrt{\mu}, 0)$ and $\mathbf{x}_{o 3}=(r-\sqrt{\mu}, 0)$ are stable agreeing well with eigenvalues given for $\mathbf{J}_{2}$ and $\mathbf{J}_{3}$.

\subsubsection{Non-linear stability: 1-parameter static bifurcation}

To determine the non-linear stability of the dynamical system we consider the use of Lyapunov methods [24]. We can use this theorem to guarantee the global stability of the system with convergence to the desired final state. The aim of the steering potential is to drive each UAV to the desired equilibrium position that corresponds to the minimum potential. Therefore, if Lyapunov's method can be used for the system, as time evolves the system will relax into the mini- 
mum energy state.

The Lyapunov function, $L$, for the system is defined in Eq. 38, where $U^{S}\left(\mathbf{x}_{i}\right)$ is given in Eq. 17;

$$
L\left(\mathbf{x}_{i}\right)=\sum_{i} U^{S}\left(\mathbf{x}_{i}\right)
$$

In order to ensure the global stability of the system the potential is defined such that the conditions given in Table 2 hold true.

Table 2: Lyapunov's Second Theorem stability conditions

\begin{tabular}{cc}
\hline \hline $\mathbf{x}_{i} \neq \mathbf{x}_{o}$ & $\mathbf{x}_{i}=\mathbf{x}_{o}$ \\
\hline$L\left(\mathbf{x}_{i}\right)>0$ & $L\left(\mathbf{x}_{o}\right)=0$ \\
$\dot{L}\left(\mathbf{x}_{i}\right)<0$ & $\dot{L}\left(\mathbf{x}_{o}\right)=0$ \\
\hline
\end{tabular}

The rate of change of the Lyapunov function can be expressed as;

$$
\frac{d L}{d t}=\sum_{i}\left[\left(\frac{\partial L}{\partial \mathbf{x}_{i}}\right) \dot{\mathbf{x}}_{i}\right]
$$

Then, substituting Eq. 24 into Eq. 52 it can be seen that;

$$
\frac{d L}{d t}=-\sum_{i} \nabla U^{s}\left(\mathbf{x}_{i}\right)^{2} \leq 0
$$

From Table 2 if $L$ is a positive definite function and $\dot{L}$ is a negative definite the system will be uniformly stable. A problem arises in the use of superimposed artificial potential functions as $\dot{L} \leq 0$. This implies that $\dot{L}$ could vanish in a position other than the goal minimum suggesting that the system may become trapped in a local minimum. In order to ensure that our system is asymptotically stable at the desired goal state the LaSalle principle [25] can be used. This extends the above constraints to state that if $L(0)=\dot{L}(0)=0$ and the set 
$\left\{\mathbf{x}_{i} \mid \dot{L}=0\right\}$ only occurs when $\mathbf{x}_{i}=\mathbf{x}_{o}$, then the goal state is asymptotically stable. Therefore, for the quadratic potential considered in this paper the LaSalle principle is valid. As we have a smooth well defined symmetric potential field, equilibrium only occurs at the goal states so the local minima problem can be avoided and the system will relax into the desired goal position.

\subsection{Hopf bifurcation - 1 parameter dynamic bifurcation}

In certain engineering applications, a formation of UAVs may be desired to form a circling surveillance pattern [2]. Frew et al. have shown how this could be achieved through the use of a Lyapunov guidance vector field approach that produces a stable convergence to a circling limit cycle behaviour for a system of UAVs [3] [26]. In bifurcation theory the Hopf bifurcation is a local bifurcation about a fixed point of a dynamical system that generates a limit cycle as the bifurcation parameter $\mu$ changes sign.

An example of such a Hopf bifurcation is given in Eq. 41 and 42 with Fig. 4 showing an example of the velocity field created when $\mu>0$. When the bifurcation parameter $\mu>0$ a pair of complex eigenvalues cross the imaginary axis and the limit cycle behaviour is induced. As $\mu$ increases the size of limit cycle also increases so that we can have a varying size of limit cycle and therefore surveillance region.

We define the Hopf bifurcation as;

$$
\begin{gathered}
\dot{x}_{i}=\mu x_{i}+y_{i}-x_{i}\left(x_{i}^{2}+y_{i}^{2}\right) \\
\dot{y}_{i}=-x_{i}+\mu y_{i}-y_{i}\left(x_{i}^{2}+y_{i}^{2}\right)
\end{gathered}
$$

The first order control model is shown in Eq. 43 with the repulsive potential added to again ensure collision avoidance and an equally spaced formation; 


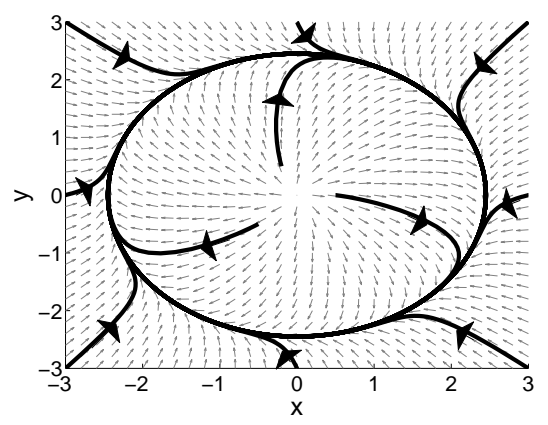

Figure 4: Hopf Bifurcation: $\mu>0$

$$
\left(\begin{array}{c}
\dot{x}_{i} \\
\dot{y}_{i} \\
\dot{z}_{i}
\end{array}\right)=\left(\begin{array}{c}
\mu x_{i}+y_{i}-x_{i}\left(x_{i}^{2}+y_{i}^{2}\right)-\frac{\partial U^{R}}{\partial x_{i}} \\
-x_{i}+\mu y_{i}-y_{i}\left(x_{i}^{2}+y_{i}^{2}\right)-\frac{\partial U^{R}}{\partial y_{i}} \\
-\alpha z_{i}-\frac{\partial U^{R}}{\partial z_{i}}
\end{array}\right)
$$

\subsubsection{Linear stability: 1-parameter dynamic bifurcation}

Similar to the analysis performed in section 2.3.1 the velocity field described by Eq. 43 is recast to determine the linear stability of the system assuming at large separation distances the repulsive potential function can be ignored. Therefore,

$$
\begin{aligned}
\left(\begin{array}{c}
\dot{x}_{i} \\
\dot{y}_{i} \\
\dot{z}_{i}
\end{array}\right) & =\left(\begin{array}{c}
\mu x_{i}+y_{i}-x_{i}\left(x_{i}^{2}+y_{i}^{2}\right) \\
-x_{i}+\mu y_{i}-y_{i}\left(x_{i}^{2}+y_{i}^{2}\right) \\
-\alpha z_{i}
\end{array}\right) \\
& =\left(\begin{array}{c}
m\left(\mathbf{x}_{i}\right) \\
n\left(\mathbf{x}_{i}\right) \\
p\left(\mathbf{x}_{i}\right)
\end{array}\right)
\end{aligned}
$$

Similarly, letting $\mathbf{x}_{o}$ denote fixed points with $\dot{x}_{i}=\dot{y}_{i}=\dot{z}_{i}=0$ so that;

$$
m\left(x_{o}, y_{o}, z_{o}\right)=0
$$




$$
\begin{aligned}
& n\left(x_{o}, y_{o}, z_{o}\right)=0 \\
& p\left(x_{o}, y_{o}, z_{o}\right)=0
\end{aligned}
$$

The Jacobian, $\mathbf{J}$, is then a $3 x 3$ matrix given by;

$$
\mathbf{J}=\left.\left(\begin{array}{ccc}
\frac{\partial}{\partial x_{i}}\left(m\left(\mathbf{x}_{i}\right)\right) & \frac{\partial}{\partial y_{i}}\left(m\left(\mathbf{x}_{i}\right)\right) & \frac{\partial}{\partial z_{i}}\left(m\left(\mathbf{x}_{i}\right)\right) \\
\frac{\partial}{\partial x_{i}}\left(n\left(\mathbf{x}_{i}\right)\right) & \frac{\partial}{\partial y_{i}}\left(n\left(\mathbf{x}_{i}\right)\right) & \frac{\partial}{\partial z_{i}}\left(n\left(\mathbf{x}_{i}\right)\right) \\
\frac{\partial}{\partial x_{i}}\left(p\left(\mathbf{x}_{i}\right)\right) & \frac{\partial}{\partial y_{i}}\left(p\left(\mathbf{x}_{i}\right)\right) & \frac{\partial}{\partial z_{i}}\left(p\left(\mathbf{x}_{i}\right)\right)
\end{array}\right)\right|_{x_{o}, y_{o}, z_{o}}
$$

Thus, it can be shown that;

$$
\mathbf{J}=\left(\begin{array}{ccc}
\mu & 1 & 0 \\
-1 & \mu & 0 \\
0 & 0 & -\alpha
\end{array}\right)
$$

The corresponding eigenvalue spectrum is therefore;

$$
\lambda_{1,2,3}=-\alpha, \mu \pm i
$$

From the eigenvalue spectrum given in Eq. 50 it can be seen that since $\mu<0$ and $\alpha>0$ the equilibrium position is linearly stable indicating a stable spiral to that position. Alternatively, if we bifurcate the system and make $\mu>0$, the eigenvalues are now either positive real or positive real with complex conjugate. Therefore, as the complex eigenvalues cross the imaginary axis at $\mu=0$ we have a bifurcation of the system from a stable spiral into the oscillatory limit cycle motion.

\subsubsection{Non-linear stability: 1-parameter dynamic bifurcation}

We can again use Lyapunov's Second Theorem to determine the non-linear stability of the system. Defining the Lyapunov function, $L$, as; 


$$
L=\frac{1}{2} \sum_{i} \mathbf{x}_{i}^{2}
$$

Therefore,

$$
\begin{gathered}
\frac{d L}{d t}=\sum_{i}\left[\frac{\partial L}{\partial x_{i}} \dot{x}_{i}+\frac{\partial L}{\partial y_{i}} \dot{y}_{i}+\frac{\partial L}{\partial z_{i}} \dot{z}_{i}\right] \\
\frac{d L}{d t}=\sum_{i}\left[\rho_{i}^{2}\left(\mu-\rho_{i}^{2}\right)-\alpha z_{i}^{2}\right]
\end{gathered}
$$

For $\mu<0$ and $\alpha>0, \dot{L} \leq 0$ so that $\rho_{i}$ is always decreasing until $L=0$ so each UAV would be attracted to the equilibrium position located at the origin $\left(x_{i}=0, y_{i}=0, z_{i}=0\right)$. Alternatively, if $\mu>0$ and $\alpha>0, \dot{L}>0$ if $\rho_{i}^{2}<\mu$ and $\dot{L}<0$ if $\rho_{i}^{2}>\mu$ so the system is attracted to a limit cycle of radius, $\rho_{i}=\mu$, in the $x-y$ plane with $z_{i}=0$.

\section{NUMERICAL RESULTS}

\subsection{Static Bifurcation Formation Patterns}

Before considering the UAV application in detail it is useful to demonstrate numerically the reconfigurable patterns and advantages of the control model developed by considering a system of agents. Depending upon the choice of the free parameters in Eq. $17\left(\mu, r, \alpha, C_{r}\right.$ and $\left.L_{r}\right)$ we can achieve three different formations; cluster, ring and double ring. Figure 5 shows the results for a swarm of 30 agents given random initial conditions with free parameters summarised in Table 3 . 


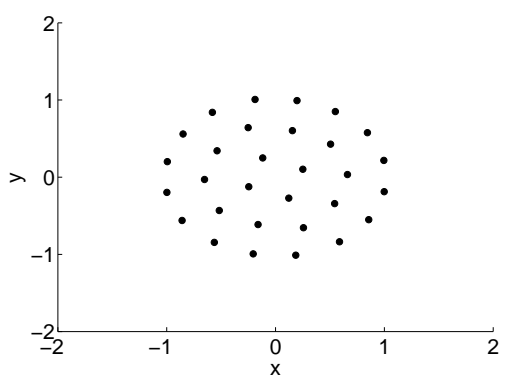

(i)

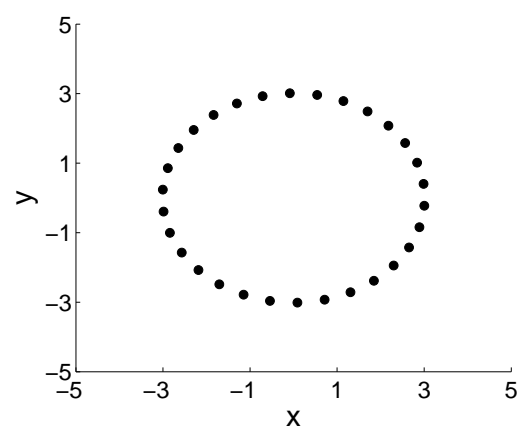

(ii)

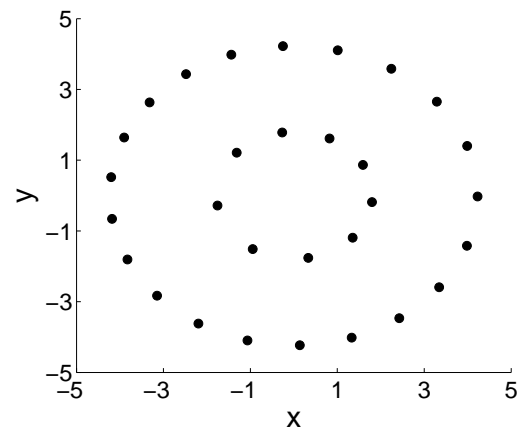

(iii)

Figure 5: Formation patterns: (i) cluster formation (ii) ring formation (iii) double ring formation 
Table 3: 1 parameter static bifurcation free parameters

\begin{tabular}{cccc}
\hline \hline Free Parameter & Cluster & Ring & Double Ring \\
\hline$\mu$ & -4 & -4 & 1.5 \\
$r$ & 0 & 3 & 3 \\
$\alpha$ & 50 & 50 & 50 \\
$z_{\text {goal }}$ & 0 & 0 & 0 \\
$C_{r}$ & 1 & 1 & 1 \\
$L_{r}$ & 0.5 & 0.2 & 0.5 \\
\hline
\end{tabular}

From the results it can be seen that the first formation corresponds to the case when $\mu<0$ and $r=0$. The agents in the system are driven towards the origin with the repulsive potential ultimately causing a uniform cluster to form. The second formation consists of a ring with radius equal to the magnitude that the pitchfork bifurcation equation is moved on the along the axis $(r=3)$. In the final formation $\mu>0$ and the stable equilibrium in the second formation becomes unstable and the system bifurcates into the double rig formation.

\subsection{Static Bifurcation Results}

Figures 6 shows the transition of a formation of 30 agents in the $x-y$ plane through the use of the static pitchfork bifurcation equation. 


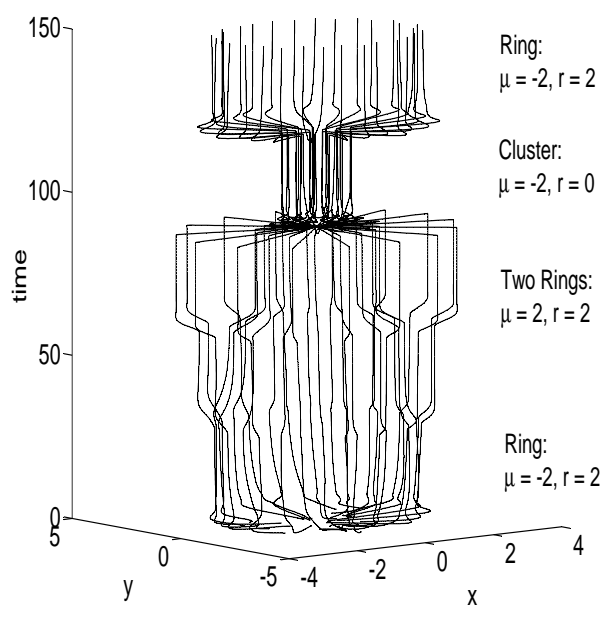

Figure 6: Transition between different formations

As it can be seen, the system bifurcates from a ring to two rings to a cluster then back to a ring. This is achieved through a simple parameter change and is one of the advantages of using the pitchfork bifurcation equation as a basis for the artificial potential function. Rather than controlling each UAV individually the global pattern of the formation can be manipulated via $\mu$.

\subsection{Hopf Bifurcation Results}

Figure 7 shows the results for a system of 15 agents interacting through the hopf bifurcation field as discussed in section 2.3. The agents in the system are given random initial conditions and required to fall onto a limit cycle behaviour with radius equal to 5 in the $x-y$ plane $(\mu=25)$. 


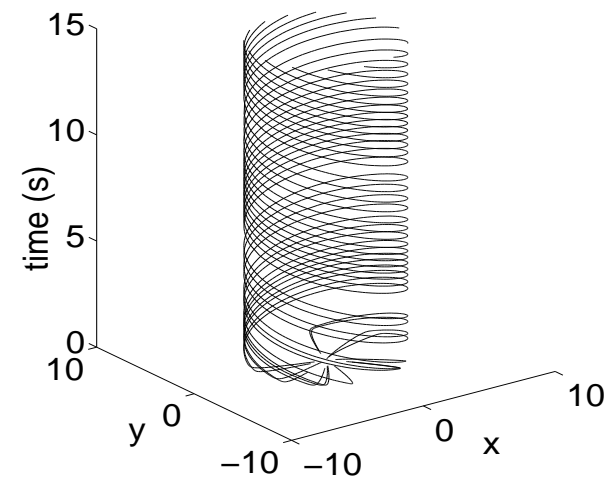

(i)

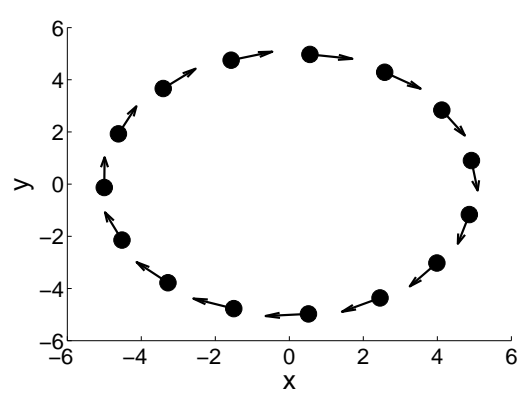

(ii)

Figure 7: Hopf bifurcation results $\left(C_{r}=1, L_{r}=2, \alpha=10, z_{\text {goal }}=0\right.$ and $\mu=25$ ) (i) time evolution (ii) final formation

As can be seen from Fig. 7 the 15 agents fall onto the desired limit cycle rotation and relaxes into constant separation formation.

\subsection{Robustness of the Model}

As one of the desirable characteristics of the model developed is that the system of agents are robust to failures it is useful to demonstrate this as shown in Fig. 8. 


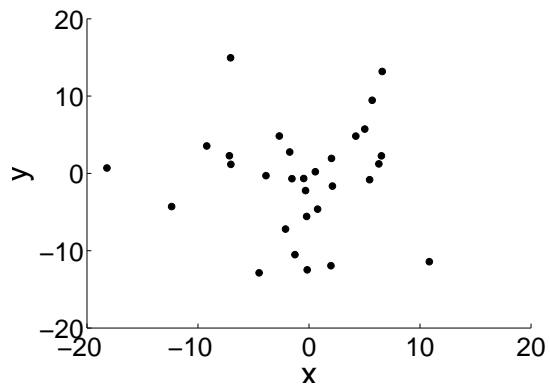

(i)

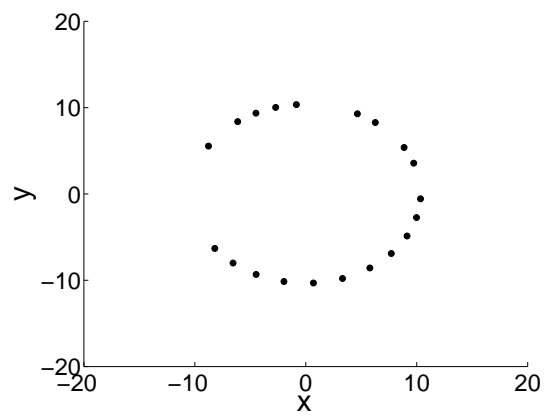

(iii)

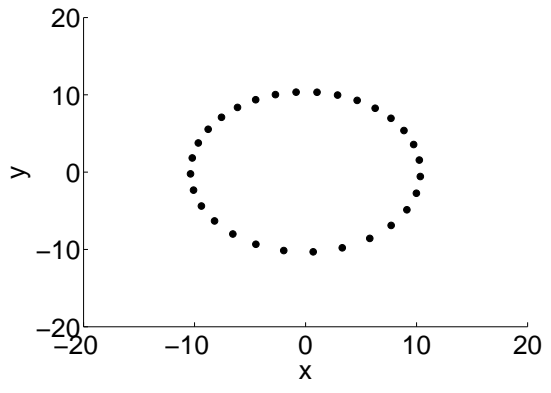

(ii)

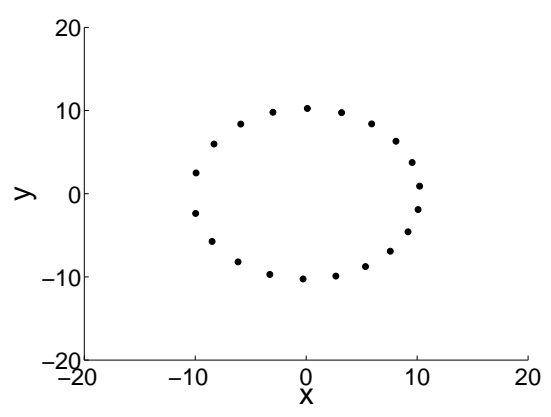

(iv)

Figure 8: Robustness of the Model (i) random initial conditions for 30 agents (ii) ring formation ( $\mu=-2, r=10, C_{r}=3$ and $L_{r}=5$ (iii) failure of 10 agents (iv) autonomous reconfiguration of the formation

As can be seen from the results a system of 30 agents fall into a ring formation with radius 10. Figure 8 (iii) shows the random failure of 10 agents with the assumption that once they fail they are completely removed from the system. The system will then autonomously reconfigure to a new ring configuration as shown in Fig. 8(iv).

\subsection{Scalable Formation}

Another advantage of the model developed is that the systems scales very well as the number of agents increase as shown in Fig. 9. 


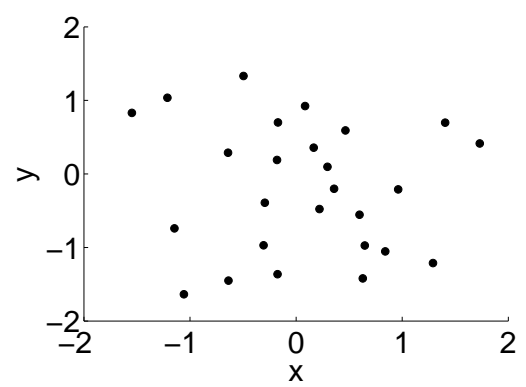

(i)

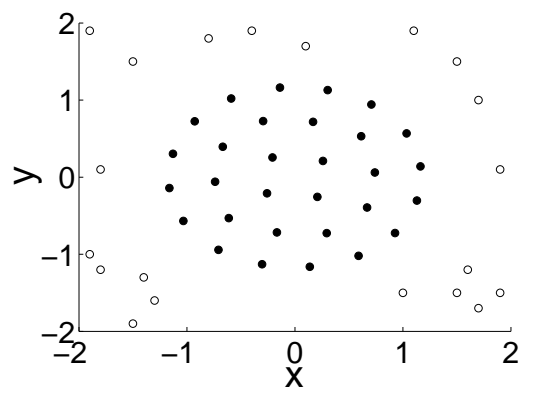

(iii)

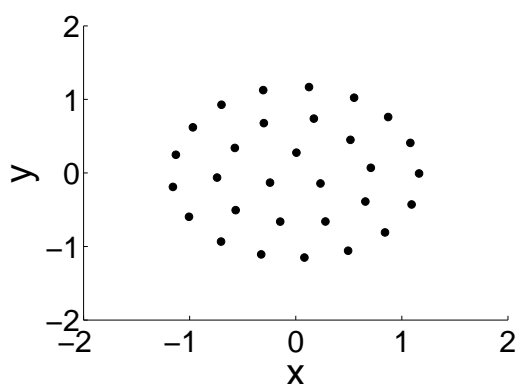

(ii)

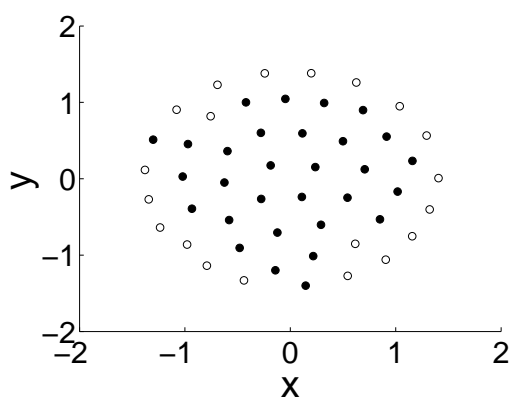

(iv)

Figure 9: Scalable formation (i) random initial conditions for 30 agents (ii) cluster formation $\left(\mu=-2, r=0, C_{r}=1\right.$ and $L_{r}=0.5$ (iii) addition of 20 agents (iv) autonomous reconfiguration of the cluster formation

Therefore, as shown in the results the system can autonomously reconfigure with the addition of new agents. 


\subsection{Flexible Formations}

The final advantage of the model is that system is flexible to obstacles and can also alter its pattern through a simple parameter change. By adding in several circular obstacles it can be shown that we can have a system of 30 agents that will create a cluster formation, autonomously manoeuver to avoid the obstacles, reconfigure into the cluster pattern and then finally bifurcate into a ring formation.

A problem with the superimposed artificial potential field method is that the system may get trapped in a local minimum as noted in section 2.3.2. To overcome this problem we consider the use of a Gaussian potential function that creates a spherical potential obstacle with no local minima as defined in Eq. $54[27]$;

$$
U_{o b s}=C_{o} \exp ^{-\left|\mathbf{x}_{i}-\mathbf{x}_{o b s}\right| / L_{o}}
$$

where, $\mathbf{x}_{o b s}$ represents the obstacle position vector and $C_{o}, L_{o}$ are the amplitude and length scale of the obstacle potential respectively.

The coordinate system is altered so that it is translating at constant speed and from the results shown in Fig. 10 it can be seen that formation of agents successfully falls into the cluster formation, manoeuvres around the obstacles reforming the cluster formation and then bifurcates into the desired ring formation. The model can therefore be considered as flexible as the formations have the ability to avoid obstacles whilst also being able to alter pattern. 


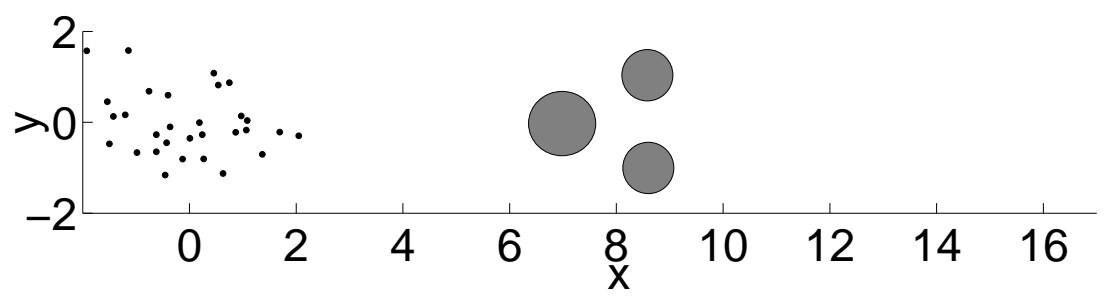

(i)

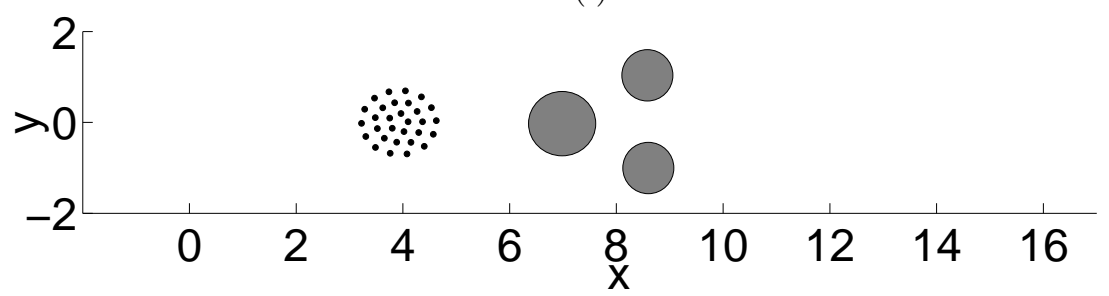

(ii)

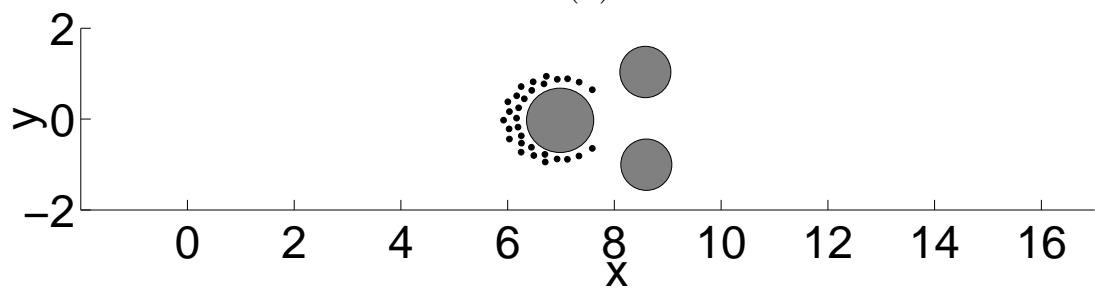

(iii)

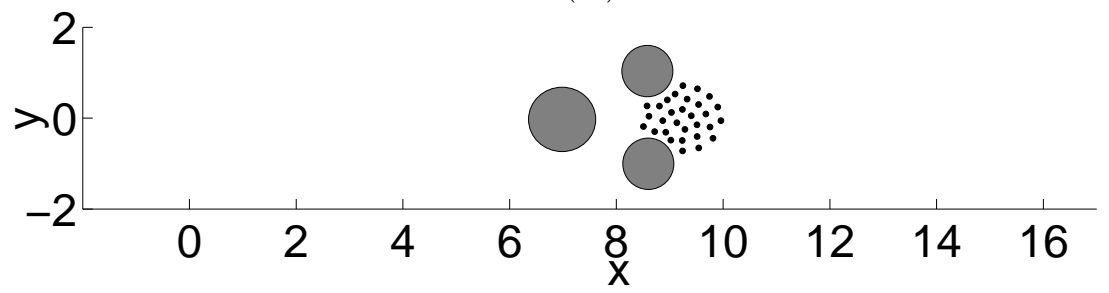

(iv)

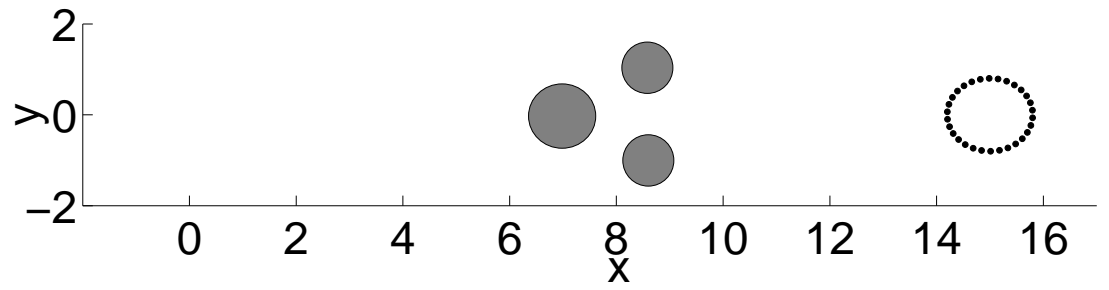

(

Figure 10: Flexible formation (i) random initial conditions (ii) cluster (8s) (iii) $\mathrm{t}=14 \mathrm{~s}(\mathrm{iv}) \mathrm{t}=18 \mathrm{~s}(\mathrm{v})$ ring formation $(30 \mathrm{~s})$ 


\section{UAV APPLICATION}

In order to test the model developed we consider a swarm of 10 commercially available Dragonfly UAV helicopters [28] that are required to fall onto a double ring formation and then bifurcate into two different ring formations in the $x-y$ plane, traveling at a final constant speed of $2.7 \mathrm{~ms}^{-1}$ whilst ensuring collision avoidance and an equally spaced final formation.

The Dragonfly X6 UAV helicopters have a cruise speed of $2.7 \mathrm{~ms}^{-1}$ and maximum turning rate defined as $\dot{\psi}_{\max }=90^{\circ} \mathrm{s}^{-1}$. It is assumed that the maximum speed and acceleration are $V_{\max }=3 m s^{-1}$ and $\dot{v}_{\max }=0.1 \mathrm{~g}$ respectively. It is also assumed that the position of the UAVs can be determined precisely and the dynamic control laws developed can accurately control the UAV state.

Although the artificial potential function method is theoretically elegant, Sigurd [29] points out that the assumption that all UAVs have information on the position of all other UAVs in the system is unrealistic as the number of UAVs increase. Each UAV will now have a sensing region that will ensure collision avoidance and an equally spaced final formation as shown in Eq. 55 and Fig. 11 , where $Z_{r}$ is the radius of repulsive zone of influence;

$$
U_{i j}^{R}=\left\{\begin{array}{ll}
\sum_{j, j \neq i} C_{r} \exp ^{-\left|\mathbf{x}_{i j}\right| / L_{r}} & \text { if }\left|\mathbf{x}_{i j}\right| \leq Z_{r} \\
0 & \text { if }\left|\mathbf{x}_{i j}\right|>Z_{r}
\end{array}\right\}
$$

The control model used is based on a simple first order velocity field tracking approach that has been used by several authors as a basic way of transforming a desired velocity field into a set of commands that control the speed and turn rate of each UAV $[1,26,30]$. The desired velocity field explained in section 2 will now be used to control each UAV by providing an actual heading 


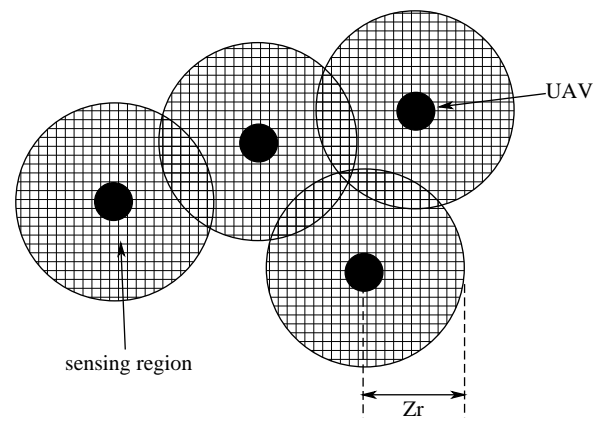

Figure 11: UAV repulsive potential sensing region

$\left(\psi_{i}\right)$ and speed $\left(V_{i}\right)$ command, assuming the formation is flying at fixed altitude.

Therefore, considering the pitchfork bifurcation described by Eq. 16 the desired velocity vectors are described by Eq. 56 and 57;

$$
\begin{gathered}
\dot{x}_{\text {desired }}=\mu \frac{x_{n}}{\rho_{n}}\left(\rho_{n}-r\right)-\frac{x_{n}}{\rho_{n}}\left(\rho_{n}-r\right)^{3}+\sum_{j, j \neq i} \frac{C_{r}}{L_{r}} \frac{x_{i j}}{\left|\mathbf{x}_{i j}\right|} \exp ^{-\left|\mathbf{x}_{i j}\right| / L_{r}}+u_{c} \\
\dot{y}_{\text {desired }}=\mu \frac{y_{i}}{\rho_{n}}\left(\rho_{n}-r\right)-\frac{y_{i}}{\rho_{n}}\left(\rho_{n}-r\right)^{3}+\sum_{j, j \neq i} \frac{C_{r}}{L_{r}} \frac{y_{i j}}{\left|\mathbf{x}_{i j}\right|} \exp ^{-\left|\mathbf{x}_{i j}\right| / L_{r}}
\end{gathered}
$$

where, as the UAVs are desired to move at a constant forward speed equal to $u_{c}$ we replace $x_{i}$ with $x_{n}=x_{i}-u_{c} t$ and $\rho_{i}$ with $\rho_{n}=\left(x_{n}^{2}+y_{i}^{2}\right)^{0.5}$ in the steering potential terms.

The desired command speed $\left(V_{\text {desired }}\right)$ and heading angle $\left(\psi_{\text {desired }}\right)$ are therefore;

$$
\begin{gathered}
V_{\text {desired }}=\left(\dot{x}_{\text {desired }}^{2}+\dot{y}_{\text {desired }}^{2}\right)^{0.5} \\
\psi_{\text {desired }}=\arctan \left(\frac{\dot{y}_{\text {desired }}}{\dot{x}_{\text {desired }}}\right)
\end{gathered}
$$

The state variables for the system are then defined as; 


$$
\left(\begin{array}{l}
x_{1} \\
x_{2} \\
x_{3} \\
x_{4}
\end{array}\right)=\left(\begin{array}{c}
V_{i} \\
\psi_{i} \\
x_{i} \\
y_{i}
\end{array}\right)
$$

A system of first order equations of motion are then solved resulting in a commanded speed and heading angle that can be used to control the UAV as shown in Eq. 61, where the constants $\lambda_{v}$ and $\lambda_{\psi}$ are inverse time constants determining the response of each $\mathrm{UAV}$;

$$
\left(\begin{array}{c}
\dot{x}_{1} \\
\dot{x}_{2} \\
\dot{x}_{3} \\
\dot{x}_{4}
\end{array}\right)=\left(\begin{array}{cc}
-\lambda_{v}\left(V_{i}-V_{\text {desired }}\right) & \text { if }\left|\dot{v}_{i}\right| \leq \dot{v}_{\max } \\
-\lambda_{\psi}\left(\psi_{i}-\psi_{\text {desired }}\right) & \text { if }\left|\dot{\psi}_{i}\right| \leq \dot{\psi}_{\max } \\
V_{i} \cos \left(\psi_{i}\right) \\
V_{i} \sin \left(\psi_{i}\right)
\end{array}\right)
$$

In addition as there is a bound on the maximum turning rate and speed there is turning circle associated with each UAV. The radius of the turning circle is defined in Eq. 62 so that if the maximum speed and turning rate are $3 m s^{-1}$ and $90^{\circ} \mathrm{s}^{-1}$ respectively, then the maximum turning radius, $R_{\text {turning }}$, is approximately $1.9 m$.

$$
R_{\text {turning }}=\frac{V_{\max }}{\dot{\psi}_{\max }}
$$

In order to estimate that size of the repulsive free parameters, $C_{r}$ and $L_{r}$, we can consider the case of 2 UAVs interacting through the repulsive potential only. Considering a simple 1-dimensional system with position coordinate, $X$, we know that for $X>>0, \frac{d X}{d t} \approx V_{\max }$. Therefore, assuming that at close separation distances the repulsive potential only acts on the UAVs we have;

$$
\frac{d X}{d t}=V_{\max }-\frac{C_{r}}{L_{r}} \exp -\frac{X}{L_{r}}
$$


The minimum separation distance, $X_{m i n}$, will therefore be estimated by setting $\frac{d X}{d t}=0$ so that

$$
X_{\min }=L_{r} \ln \left(\frac{C_{r}}{V_{\max } L_{r}}\right)
$$

In order to ensure collision avoidance, the minimum separation distance between the UAVs in the formation must be $2 \times R_{\text {turning }}=3.8 \mathrm{~m}$. The repulsive potential constants, $C_{r}$ and $L_{r}$, are therefore chosen to ensure that the minimum separation, $X_{\min }$, is greater than this value. Each UAV is given random initial conditions in the $x-y$ plane with initial speed of $2.7 \mathrm{~ms}^{-1}$ and random initial heading angles. The free parameter values are summarised in Table 4.

Table 4: 1 parameter static bifurcation free parameters

\begin{tabular}{ccccccccc}
\hline \hline formation & time $(\mathrm{s})$ & $\mu$ & $r$ & $C_{r}$ & $L_{r}$ & $\lambda_{v}$ & $\lambda_{\psi}$ & $Z_{R}$ \\
\hline double ring & $0-200$ & 100 & 30 & 73 & 20 & 1 & 1 & 45 \\
ring & $200-400$ & -1 & 40 & 73 & 10 & 1 & 1 & 45 \\
ring & $400-600$ & -1 & 50 & 73 & 10 & 1 & 1 & 45 \\
\hline
\end{tabular}

From the results shown in Fig. 12, 13 and 14 it can be seen that the formation successfully creates the desired equally spaced double ring formation and bifurcates into the two ring formations. Figure 12 shows the final formation, whereas Fig. 13 shows the time evolution for each formation. It can also be seen from Fig. 14 that the formation relaxes into a constant separation distance for each formation and that collision avoidance is ensured throughout the simulation. 

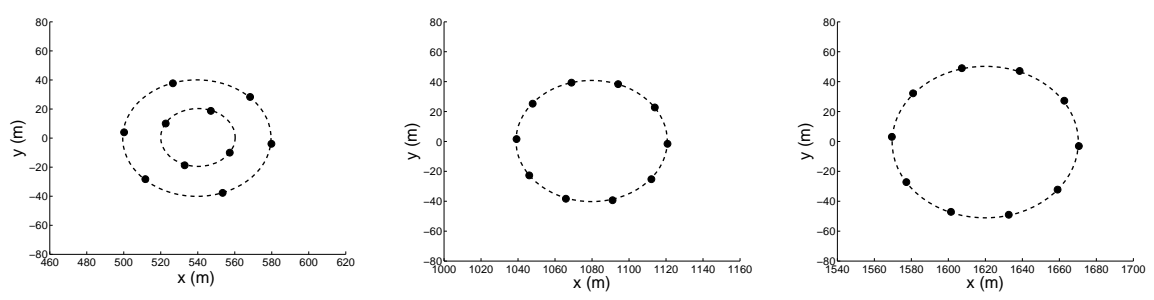

Figure 12: UAV formations in final state (i) double ring (ii) ring (radius $=40 \mathrm{~m}$ (iii) ring (radius $=50 \mathrm{~m}$ )

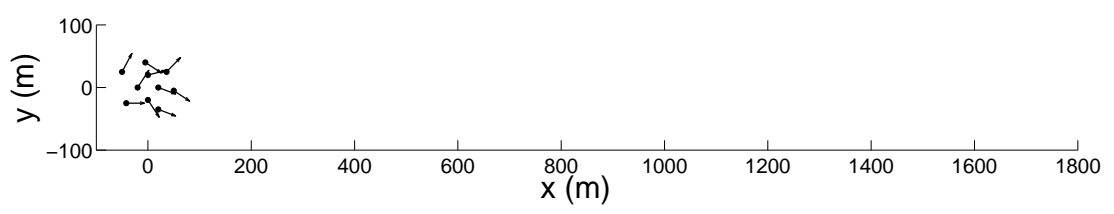

(i)

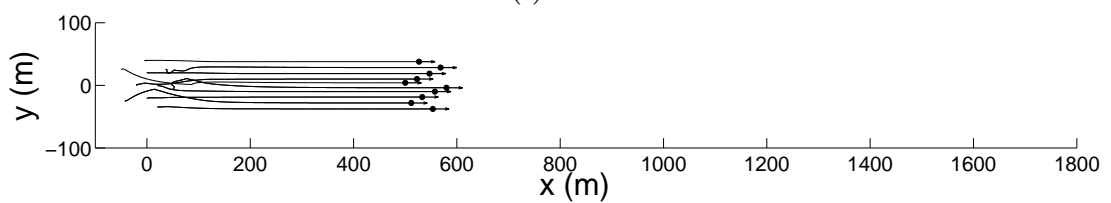

(ii)

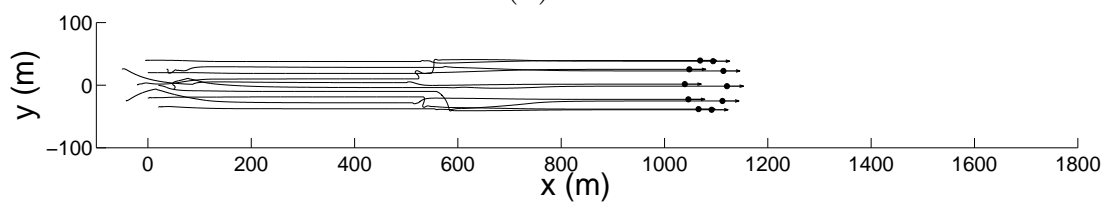

(iii)

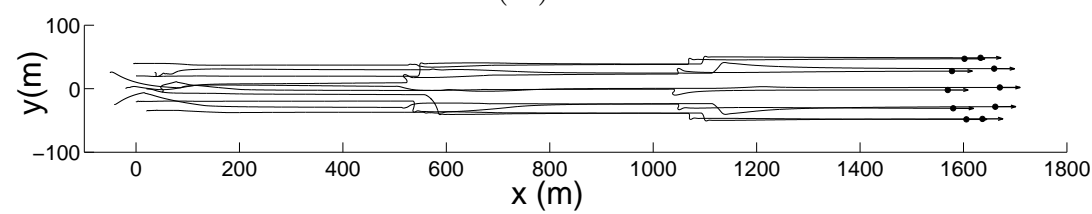

(iv)

Figure 13: UAV results (i) random initial conditions (ii) formation after $200 s$ (iii) formation after $400 \mathrm{~s}$ (iv) final desired ring formation after $600 \mathrm{~s}$ traveling at constant speed 


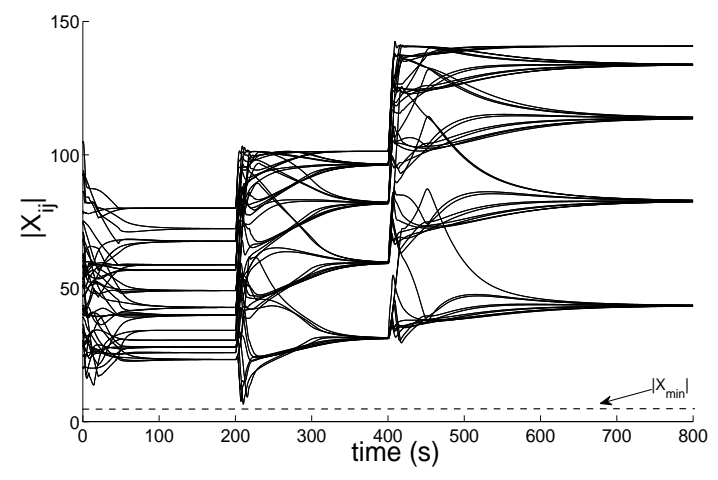

Figure 14: UAV separation: indicating an equally spaced final formation with collision avoidance

Therefore, through the use of a velocity field tracking approach we are able to generate a real set of commands that can control a swarm of UAVs, allowing a reconfigurable equally spaced formation with collision avoidance assured.

\section{CONCLUSION}

It has been shown how a behavioural based control method can be used to create various patterns for a formation of UAVs and has the advantages of being robust, scalable and flexible. Dynamical systems theory is used as the basis of the control method with the new approach of bifurcating potential fields used for pattern formation and reconfigurability. A first order velocity field is used to command each UAV based on a steering and repulsive potential field. It is shown that there exists a scale separation between the steering and repulsive potential so that each UAV moves under the influence of a long range steering potential but with short range collision avoidance. Using this the stability of the system was demonstrated analytically to ensure that desired behaviours always occur. To demonstrate the algorithm developed we consider the control of $10 \mathrm{UAVs}$ that are required to form an equally spaced double ring formation and then 
bifurcate into two different ring formations traveling at constant speed whilst also ensuring collision avoidance. A simple 1st order velocity field tracking approach is used to track the desired velocity field and generate a set a real commands that control the aerodynamic surfaces of the UAV. We consider the use of 10 commercially available UAV helicopters that have maximum turning rate of $90^{\circ} \mathrm{s}^{-1}$. The numerical results successfully show the formation of an equally spaced double ring of UAVs that then bifurcates into two different ring formations, traveling at constant speed with collision avoidance ensured.

\section{References}

[1] C. R. McInnes. Velocity field path-planning for single and multiple unmanned aerial vehicles. The Aeronautical Journal, 107(1073):419-426, 2003 .

[2] R. Beard, D. Kingston, M. Quigley, D. Snyder, R. Christiansen, W. Johnson, T. McLain, and M. Goodrich. Autonomous vehicle technologies for small fixed wing UAVs. In 2nd AIAA Unmanned Unlimited Systems, Technologies, and Operations, San Diego, California, 15 - 18 September 2003.

[3] E. Frew, D.A. Lawrence, and S. Morris. Coordinated standoff tracking of moving targets using lyapunov guidance vector fields. Journal of Guidance, Control and Dynamics, 31(2):290-306, March-April 2008.

[4] R. Teo, J.S Jang, and C.J. Tomlin. Automated multiple UAV flight - the stanford dragonfly UAV program. In 43rd IEEE Conference on Decision and Control, Atlantis, Paradise Island, Bahamas, 14 - 17 December 2004.

[5] M. Quigley, M.A. Goodrich, S. Griffiths, A. Eldredge, and R.W. Beard. Target acquisition, localization, and surveillance using a fixed-wing miniUAV and gimbaled camera. In IEEE International Conference on Robotics and Automation, pages 2600-2605, 18-22 April 2005. 
[6] A. Ollero and L. Merino. Control and perception techniques for aerial robotics. Annual Reviews in Control, 28(2):167-178, October 2004.

[7] O. Ilaya, C. Bil, and M. Evans. Control design for unmanned aerial vehicle swarming. Proc. of the Institution of Mechanical Engineers, Part G: Journal Aerospace Engineering, 222(4):549-567, 2008.

[8] R. A. Brooks. A robust layered control system for a mobile robot. IEEE Journal Of Robotics And Automation, 2(1):14-23, 1986.

[9] T. Balch and R.C. Arkin. Behavior-based formation control for multi-robot teams. IEEE Transaction on Robotics and Automation, 14(6):926-939, December 1998.

[10] J.H. Reif and H. Wang. Social potential fields: A distributed behavioral control for autonomous robots. Robots and Autonomous Systems, 27(3):171-194, 1999.

[11] K. Han, J. Lee, and Y. Kim. Unmanned aerial vehicle swarming control using potential functions and sliding mode control. Proc. of the Institution of Mechanical Engineers, Part G: Journal Aerospace Engineering, 222(6):721-730, 2008.

[12] O. Khatib. Real-time obstacle avoidance for manipulators and mobile robots. The International Journal of Robotics Research, 5(1):90-98, 1986.

[13] A Badawy and C.R. McInnes. Robot motion planning using hyperboloid potential functions. In World Congress on Engineering, London, UK, July 2-4 2007.

[14] S.S. Ge and Y.J Cui. Dynamic motion planning for mobile robots using potential field method. Autonomous Robots, 13(3):207-222, November 2002.

[15] D.J. Bennet and C.R. McInnes. Pattern transition in spacecraft formation flying via the artificial potential field method and bifurcation theory. In 3rd 
International Symposium on Formation Flying, Missions and Technologies, Noordwijk, The Netherlands, April 23-25, 2008.

[16] A. Badawy and C.R. McInnes. On-orbit assembly using superquadric potential fields. Journal of Guidance, Control, and Dynamics, 31(1):30-43, 2008.

[17] D. Izzo and L. Pettazi. Autonomous and distributed motion planning for satellite swarm. Journal of Guidance, Control, and Dynamics, 30(2):449$459,2007$.

[18] W.J. Crowther. Rule-based guidance for flight vehicle flocking. Proc. of the Institution of Mechanical Engineers, Part G: Journal Aerospace Engineering, 218(2):111-124, April 2004.

[19] P. Basu, J. Redi, and V. Shurbanov. Coordinated flocking of UAVs for improved connectivity of mobile ground nodes. In IEEE Military Communications Conference, volume 3, 31 Oct - 3rd Nov 2004.

[20] R. Olfati-Saber. Flocking of multi-agent dynamic systems: Algorithms and theory. IEEE Transactions of Automation Control, 51(3):401-420, March 2006.

[21] K.H. Tan and M.A. Lewis. Virtual structures for high precision cooperative mobile robotic control. In IEEE/RSJ International Conference Intelligent Robots and Systems, volume 1, pages 132-139, Osaka, Japan, 4-8 November 1996.

[22] M. R D'Orsogna, Y. L Chuang, A. L Bertozzi, and S. Chayes. Self-propelled particles with soft-core interactions: patterns, stability and collapse. Physical Review Letters, 96(10):14302-1 - 14302-4, March 2006.

[23] D.W. Jordon and P. Smith. Nonlinear Ordinary Differential Equations: An Introduction to Dynamical Systems. Oxford University Press, 1999. 
[24] R. E. Kalman and R. E. Bertram. Control system analysis and design via the second method of lyapunov: (i) continuous-time systems (ii) discrete time systems. Transactions ASME Journal Basic Engineering, 82:371-393, June 1960.

[25] J.P. LaSalle. An invariance principle in the theory of stability. International Symposium on Differential Equations and Dynamical Systems, pages 277286, 1967. Ed. New York: Academic Press.

[26] D.A. Lawrence, E. Frew, and J. Pisano. Lyapunov vector fields for autonomous unmanned aircraft flight control. Journal of Guidance, Control and Dynamics, 31(5):1220-1229, September-October 2008.

[27] F. McQuade. Autonomous control for on-orbit assembly using artificial potential functions. PhD thesis, University of Glasgow, 1997.

[28] Dragonfly X6 UAV RC Helicopter. http://www.draganfly.com/uavhelicopter/draganflyer-x6/, 18th December 2008.

[29] K. Sigurd and How. J. UAV trajectory design using total field collision avoidance. In AIAA Guidance, Navigation and Control Conference, August 2003.

[30] G. Gowtham and K. S. Kumar. Simulation of multi UAV flight formation. In 24th Digital Avionics Systems Conference, volume 2, 30th October - 3rd November 2005. 